\title{
Caracterización Psicosocial de las Familias del Barrio 20 de Julio Municpio de Urrao Antioquia
}

\author{
Psychosocial Characterization of the Families of the \\ "20 de Julio" Neighborhood, Municipality of Urrao, Antioquia.

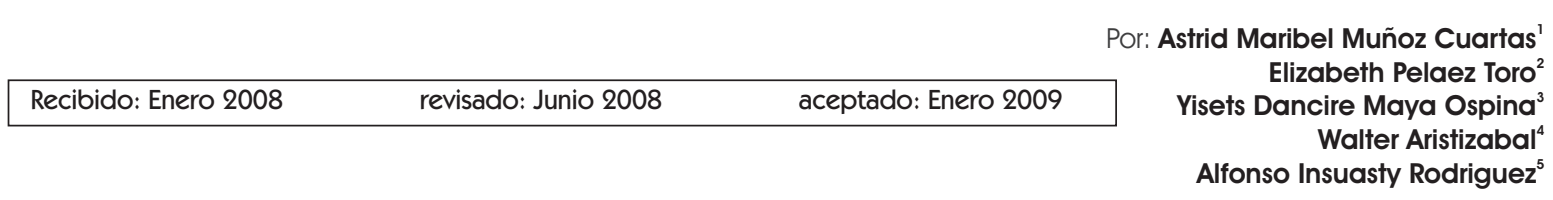

\section{Resumen:}

Este artículo presenta el resultado de una Investigación, realizada por la Universidad de San Buenaventura Medellín en el Barrio 20 de Julio del Municipio de Urrao (Antioquia). El estudio fue de tipo descriptivo-retrospectivo; en éste participaron familias y jóvenes, además de cinco instituciones que han prestado atención directa a la comunidad. El propósito del estudio fue caracterizar la población del barrio, con el fin de identificar los factores actuales que afectan la convivencia. Los resultados descritos en la caracterización de las familias se agrupan en: características socio demográficas, tiempo de residencia en el barrio, lugar de procedencia de la familia y razones que llevaron a las familias a habitar el barrio, composición y dinámica familiar, condiciones de la vivienda en general y percepción de la familia sobre las problemáticas que afectan el barrio. Finalmente se presenta la discusión y las conclusiones sobre los hallazgos.

\section{Palabras Claves:}

Estructura familiar, dinámica familiar, estilos parentales, factores de convivencia, características sociodemográficas, percepción de problemáticas. .

\section{Abstract:}

This article shows the results of a research carried out by Saint Bonaventure University, Medellin, in the "20 de Julio" Neighborhood in the Municipality of Urrao, Antioquia. It was a descriptive-retrospective study in which families and young people took part, as well as five institutions which have served directly to the community.

The purpose of the study was to characterize the population of the neighborhood, in order to identify the current factors which interfere with cohabitation.

The results described in the characterization of the families are grouped into: sociodemographic characteristics, time of living in the neighborhood, place of origin, and the reasons which led these families to live in the neighborhood, family make-up and dynamic, the housing conditions in general and the perception the family has about the problems which affect the neighborhood. Lastly, a discussion and the conclusions about the findings are presented.

\section{Key words:}

Family structure, family dynamics, parental styles, cohabitation factors, socio-demographic characteristics, perception of problems. 


\section{Introducción}

La situación de conflicto ideológico-político armado que ha vivido el país, y especialmente el Departamento de Antioquia en los últimos veinte años, ha desencadenado diversos efectos en la población civil (Estrada, Ibarra y Sarmiento, 2003; Rubio, 1997), especialmente en ciertos contextos territoriales subregionales como es el caso del Suroeste Antioqueño.

En este sentido, Urrao, municipio perteneciente a la región del Suroeste Antioqueño, es elegido de entre 55 municipios que fueron considerados por la Gobernación de Antioquia, como territorio de alta conflictividad ciudadana, problemática ésta, frente a la que debería aplicarse una estrategia de seguridad ciudadana y justicia, que iniciará con la formulación e implementación de un modelo para la construcción colectiva de la seguridad y crear condiciones para la convivencia y la paz. Así, con el apoyo del Departamento de Formación Humana y Bioética de la Universidad de San Buenaventura de Medellín y el grupo de investigación Democracia, Paz y Desarrollo, perteneciente a éste departamento, se realizó la caracterización de una comunidad barrial del municipio de Urrao, como primera fase para la aplicación de una propuesta de intervención en comunidades altamente afectadas por el conflicto armado interno. El barrio seleccionado por la Asesoría de Paz de la Gobernación de Antioquia, fue el "Barrio 20 de Julio", por ser el territorio urbano en el que se han identificado el mayor número de reportes de acciones violentas contra la ciudadanía.

En esa dirección el interés del presente artículo, es el de presentar el segundo avance de los resultados obtenidos en la investigación realizada en el "Barrio 20 de Julio", centrándose principalmente en la caracterización realizada a las familias. En primer lugar, se presentan

\footnotetext{
'Candidata a Magíster en Educación Universidad San Buenaventura Medellín. Psicóloga Universidad de San Buenaventura Medellín. Miembro del grupo de investigación Democracia, Paz y Desarrollo del Departamento de Formación Humana y Bioética de la Universidad de San Buenaventura Medellín. mary.munozc@gmail.com ${ }^{2}$ Candidata a Magíster en Educación Universidad San Buenaventura Medellín. Psicóloga Universidad de Antioquia. Miembro del grupo de investigación Democracia, Paz y Desarrollo del Departamento de Formación Humana y Bioética de la Universidad de San Buenaventura Medellín. elizabethpelaeztoro@yahoo.es

${ }^{3}$ Estudiante en la especialización Evaluación Pedagógica en la Universidad Católica de Manizales. Psicóloga Universidad de Antioquia. yisdanmaos@yahoo.es

${ }^{4}$ Candidato a Magíster en Educación Universidad CINDE. Antropólogo Universidad de Antioquia. Miembro del grupo de investigación Democracia, Paz y Desarrollo del Departamento de Formación Humana y Bioética de la Universidad de San Buenaventura Medellín. waristis@yahoo.com

${ }^{5}$ Docente Investigador Universidad de San Buenaventura Medellín. Director del grupo de investigación Democracia, Paz y Desarrollo del Departamento de Formación Humana y Bioética de la Universidad de San Buenaventura Medellín. alfonso.insuasty@gmail.com
} 
algunos referentes conceptuales. Como segundo, se realiza la descripción de la metodología propuesta para el estudio. En el tercer punto, se exponen los resultados encontrados que caracterizan la composición y dinámica familiar y por último se expone la discusión y conclusiones de los hallazgos.

\section{Referentes teóricos}

\subsection{Definición de familia}

Para la palabra familia no existe un vocablo etimológico exacto, sin embargo, se le ha asociado con el término latín fames (hambre) y con el palabra famulus (sirviente), indicando que en sus orígenes el concepto de familia estaba referido al conjunto de esclavos y criados propiedad de un solo hombre.

Según Hernández (1997), en la actualidad la familia se define como un sistema natural y evolutivo, alrededor del cual giran otras concepciones como: institución social, que tiene como fin transmitir reglas de comportamiento; grupo que se organiza para preservar la supervivencia; construcción cultural, que se apropia de unas tradiciones, pero que en ese intercambio con su ambiente exterior reforma la cultura, reformula la tradición y cambia la sociedad; y como conjunto de relaciones emocionales, donde los miembros satisfacen las necesidades emocionales y practican las emociones.

De acuerdo con estas concepciones, Quintero (1997) atribuye a la familia las siguientes funciones:

La protección psicosocial de sus miembros, la cual engendra nuevas personas y responde por el desarrollo integral de todos sus miembros.

La inserción del individuo en su cultura y su transmisión, lo que se conoce como la socialización.

\subsection{Tipologías de familia}

Siguiendo a Quintero (1997), las tipologías hacen referencia a la forma en que las familias se organizan teniendo en cuenta los miembros que la constituyen y el tipo de lazos que los unen. Para efectos del presente artículo sólo se referencian aquellas que fueron identificadas en el Barrio 20 de Julio. Estas son: 
Familia nuclear: compuesta por dos adultos con sus respectivos hijos. Unidos por lazos de consanguinidad y viven bajo el mismos techo.

Familia con un solo progenitor, monoparental o uniparental: en ella viven los hijos sólo con el padre o con la madre en situación de soltería, divorcio o viudez.

Familia Extensa: en ella pueden vivir la pareja con o sin hijos, y otros miembros como sus parientes consanguíneos (abuelos, tíos, primos y otros parientes), junto a la familia nuclear.

Estructura unipersonal, ciclo individual u hogar unipersonal: corresponde a personas que viven solas sin compartir la vivienda. Es frecuente en las personas solteras viudas y adultos mayores.

\subsection{Dinámica Familiar}

La dinámica familiar se refiere a la manera en que los miembros se relacionan en cuanto a aspectos como: tipo de apego, comunicación, límites, roles, toma de decisiones y manera de resolver los conflictos (Hernández, 1997; Quintero 1997).

El apego es la tendencia a establecer lazos emocionales íntimos cuya función propia y clave es la supervivencia y protección (Bowlby, 1993). La familia se convierte en el lugar en el cual las personas establecen relaciones de apego y compromiso emocional, de importancia porque éste influye en el funcionamiento efectivo de la personalidad y de la salud mental, es decir, tiene una incidencia en el desarrollo del si mismo, los autoesquemas, el proceso de individuación y el establecimiento las relaciones afectivas con otras personas.

La comunicación por su parte, es un factor de importancia para el desarrollo de las relaciones familiares, porque se convierte en una de las maneras de mediar las tensiones y proporcionar oportunidades para que sus miembros expresen sus ideas, emociones y estados de ánimo. Para que la comunicación contribuya en el funcionamiento óptimo de la familia, es necesario que sea asertiva, esto es positiva, clara, directa, continua y enriquecedora.

De acuerdo con Hernández (1997), los límites, los roles y las normas influyen en la manera como se organiza la dinámica familiar. Los límites componen el perímetro del sistema familiar; cuando éstos son excesivamente permeables la familia puede perder su identidad e integridad. Por el contrario cuando son escasos, el sistema se cierra y se aísla. Sobre los roles y reglas, la autora señala que pueden ser implícitos o explícitos y contribuyen en la organización de las responsabilidades e interacción familiar, además ayudan en la prescripción y limites de la conducta de los miembros para mantener la estabilidad del grupo (Hernández, 1997). 
Dentro de la dinámica familiar es importante rescatar el concepto de tensión familiar, por ser un elemento propio en la interacción cotidiana, ésta puede ser comprendida como una fuerza específica del sistema familiar que tiende a distorsionar y puede ser manifiesta u oculta, aislada o habitual, permanente o temporal, real o imaginaria, universal o especifica (Quintero, 1997).

Según Quintero (1997), las tensiones pueden estar en relación con crisis que afectan a las familias como la muerte de un ser querido, dificultades económica, migraciones o desplazamiento, invalidez física o mental, calamidades naturales, procesos de ruptura, familias violentas, familias con miembros suicidas o farmacodependientes, familias que infringen la ley permanentemente, entre otras. La manera de resolver las tensiones habla de los recursos familiares para hacer frente a las crisis, los cuales pueden dividirse en recursos que contribuyen a mejorar la situación, a agravarla, mantenerla, evitarla o no resolverla.

Por ultimo es importante señalar que la desviación de la salud individual y familiar depende del equilibrio entre esfuerzos, recursos y necesidades, de igual manera, la disfunción familiar es un factor predisponente de aparición de dificultades psicosociales (Hernández, 1997).

\subsection{Estilos parentales}

Los estilos parentales, pueden comprenderse como constelación o patrones de prácticas, actitudes y tácticas paternas habituales en la interacción entre padres e hijos, los cuales han recibido diversos nombres: estrategias de socialización, estilos educativos paternos, estilos paternos de socialización, estilos parentales, entre otros (Esteve, 2004). Desde la pedagogía Coloma (1993) los define como "esquemas prácticos que reducen las múltiples y minuciosas pautas educativas paternas a unas pocas dimensiones básicas, que cruzadas entre sí en diferentes combinaciones, dan lugar a diversos tipos habituales de educación familiar".

Para el caso de esta investigación se emplea la nominación de estilos parentales, con la clasificación realizada por Baumrid (1989), autor que propone la existencia de dos dimensiones subyacentes en las relaciones paterno-filiales: la aceptación y el control parental, a partir de las cuales identifican tres estilos básicos de control parental, cualitativamente diferentes: democrático, autoritario y permisivo (Baumrid, 1989). Se agrega además el negligente de la clasificación de Musitu (1992, 1994).

Estilo parental democrático (authoritative): también llamado asertivo, se caracteriza por la existencia de una alta implicación y alta supervisión por parte de los padres, sin embargo ésta se da en un punto intermedio, controlando a los hijos por medio de la razón más que por la imposición. 
Estilo parental permisivo: existencia de una alta implicación y baja supervisión por parte de los padres, no se ejerce prácticamente ningún control con el grado máximo de autonomía.

Estilo parental autoritario: se caracteriza por una baja implicación y alta supervisión de los padres. Se valora sobre todo la obediencia y se limita y restringe el grado de autonomía.

Estilo parental negligente: hay una baja implicación y baja supervisión por parte de los padres, es característico por la escasa aceptación del hijo, poca implicación en su conducta y bajo nivel de coerción e imposición. Son indiferentes con la conducta del hijo tanto sea buena o mala, no dialogan, son poco afectivos y se implican poco en su educación.

\subsection{Definición de vivienda y calidad de vivienda}

Según UNICEF (2001), "la vivienda es un lugar donde se socializa con los demás. La cultura de cualquier grupo está anclada en un modelo de vida hogareña y se configura por el eterno material". En éste sentido la vivienda debe cumplir con unos requisitos mínimos de infraestructura y servicios públicos que garanticen la calidad de vida de las personas.

De acuerdo con el Pacto Internacional Sobre Derechos Económicos, Sociales y Culturales existen siete parámetros básicos para el reconocimiento de la vivienda adecuada (Terre DESC Hemmes Suisse, Universidad de San Buenaventura, 2008), a saber:

* Seguridad jurídica a la tenencia

Disponibilidad de servicios, materiales, instalaciones e infraestructura

* Accesibilidad financiera (gastos soportables)

* Carácter de habitabilidad

* Asequibilidad (cercanía al equipamiento público, y conexión con las redes de comunicación de la ciudad o el municipio)

* Ubicación (lugar). Proximidad a los servicios públicos, recreación, entre otros; proxemia con los vecinos acceso a los sitios de trabajo. Seguridad en la estabilidad y accesibilidad a las casas

* Adecuación cultural

\section{Metodología aplicada}

En la presente investigación se realizo un estudio descriptivo retrospectivo con el fin de identificar los factores de "riesgo y de protección" involucrados en el tema de convivencia y paz en el Barrio 20 de Julio del Municipio de Urrao. 


\subsection{Participantes}

La población objeto de estudio estuvo comprendida por una muestra de 90 familias que participaron de manera voluntaria y que durante el año 2008 estuvieron viviendo en el Barrio 20 de Julio del Municipio de Urrao-Antioquia,

\subsection{Procedimiento}

Dado el objeto de estudio se optó por una metodología cuanti-cualitativa, en la que se emplearon fuentes de información primarias y secundarias. Entre las primeras, se empleó el Instrumento Caracterización Familiar, dirigido a las personas cabeza de familia dividido en 7 partes: datos socioeconómicos, composición y dinámica familiar, problemáticas generales y relevantes del grupo familiar, antecedentes familiares, redes de apoyo familiares, condiciones de vivienda y percepción de la familia sobre el barrio. Para el caso de las fuentes de información secundarias se tuvieron en cuenta: observación directa, talleres con la comunidad, entrevistas abiertas y conversatorios.

Para el procesamiento de los datos, se empleó el programa Epi Info 2000, en el cual se tabuló la información recolectada, además de almacenar los datos se realizaron los cálculos correspondientes para los análisis univariados, conformados por estadísticos descriptivos a partir de los que se generaron frecuencias absolutas y relativas para las variables de interés. La información cualitativa fue consignada en diarios de campo y fue posteriormente empleada para dar soporte a los datos cuantitativos.

\section{Resultados}

\subsection{Caracterización sociodemográfica}

La información corresponde a 90 familias encuestadas pertenecientes al Barrio 20 de Julio del Municipio de Urrao- Antioquia. El total de personas que conforman las familias encuestadas corresponde a 418. 


\subsubsection{Total de personas por familia, edad y género}

\section{Gráfico 1-. Distribución de personas que conforman el grupo familiar.}

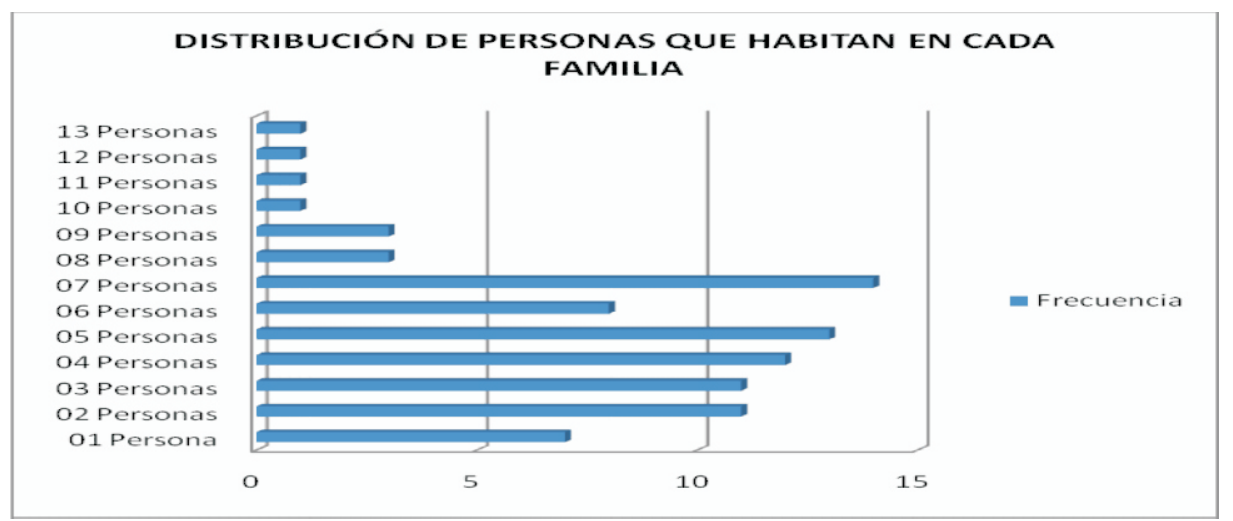

Fuente: Base de datos Proyecto: Caracterización psicosocial de las familias del Barrio 20 de JulioMunicipio de Urrao.

El número de personas que conforman los grupos familiares oscilan entre 1 y 13 miembros; la gráfica 1 indica que la mayoría de las familias están compuestas entre 2 y 7 personas, siendo 7 el grupo con el mayor porcentaje.

\section{Tabla 1. Total de mujeres y hombres de acuerdo al grupo etareo}

\begin{tabular}{|l|c|c|c|c|c|c|}
\hline \multicolumn{1}{|c|}{ Edad mujer } & Frecuencia & Porcentaje & \multicolumn{2}{c|}{ Edad hombre } & Frecuencia & Porcentaje \\
\hline No responde & 0 & $0,0 \%$ & No responde & 0 & $0,0 \%$ \\
\hline a. 0 -5 años & 45 & $10,7 \%$ & a. 0 -5 años & 50 & $11,9 \%$ \\
\hline b. 6 -12 años & 30 & $7,1 \%$ & b. $6-12$ años & 30 & $7,1 \%$ \\
\hline c. 13-17 años & 55 & $13,1 \%$ & c. 13- 17 años & 41 & $9,8 \%$ \\
\hline d. 18 años en adelante & 87 & $20,8 \%$ & d. 18 años en adelante & 80 & $19,1 \%$ \\
\hline \multicolumn{1}{|c|}{ Total } & $\mathbf{2 1 7}$ & $\mathbf{5 1 , 7 \%}$ & Total & $\mathbf{2 0 1}$ & $\mathbf{4 7 , 9 \%}$ \\
\hline
\end{tabular}

Fuente: Base de datos Proyecto: Caracterización psicosocial de las familias del Barrio 20 de Julio Municipio de Urrao. 
La distribución por grupos de edad, refleja que en las familias el mayor porcentaje son personas mayores de 18 años (40\%), seguido por los infantes menores de 5 años (23\%) y en igual proporción se encuentran los jóvenes entre 13 y 17 años (23\%). Se observa en términos generales que, de acuerdo a los grupos etareos la población se distribuye de manera homogénea.

\subsubsection{Nivel educativo de la familia}

Tabla 2. Grado de escolarización de las personas que no estudian actualmente

\begin{tabular}{|c|c|c|}
\hline \multirow{2}{*}{ Nivel de escolaridad } & \multicolumn{2}{|c|}{ Personas } \\
\hline & Frecuencia & $\%$ \\
\hline Analfabeta & 55 & $13 \%$ \\
\hline Primaria Incompleta & 103 & $24.4 \%$ \\
\hline Primaria Completa & 34 & $7.9 \%$ \\
\hline Secundaria Incompleta & 33 & $7.7 \%$ \\
\hline Secundaria Completa & 18 & $4.1 \%$ \\
\hline Técnico & 0 & $0 \%$ \\
\hline Tecnológico & 0 & $0 \%$ \\
\hline Universitario & 0 & $0 \%$ \\
\hline Población en edad no escolar & 68 & $16.3 \%$ \\
\hline Total & 243 & $57.5 \%$ \\
\hline
\end{tabular}

Fuente: Base de datos Proyecto: Caracterización psicosocial de las familias del Barrio 20 de Julio-Municipio de Urrao.

La tabla 2 presenta el grado de escolaridad de las personas que no estudian actualmente, se destaca que el $7.9 \%$ de esta población ha concluido sus estudios primarios y el $4.1 \%$ sus estudios de bachillerato. No se reportan casos con grados superiores a la básica secundaria y el $16.3 \%$ corresponde a las población en edad no escolar. 


\section{Tabla 3. Grado de escolarización de personas que estudian actualmente}

\begin{tabular}{|c|c|c|}
\hline \multirow{2}{*}{$\begin{array}{c}\text { Nivel de escolaridad de } \\
\text { personas que estudian } \\
\text { actualmente }\end{array}$} & \multicolumn{2}{|c|}{ Personas } \\
\hline & Frecuencia & $\%$ \\
\hline Analfabeta & $\mathrm{O}$ & $0,0 \%$ \\
\hline Primaria & 63 & $15 \%$ \\
\hline Secundaria & 44 & $10.8 \%$ \\
\hline Técnico & $\mathrm{O}$ & $0 \%$ \\
\hline Tecnológico & $\mathrm{O}$ & $\mathrm{O} \%$ \\
\hline Universitario & $\mathrm{O}$ & $\mathrm{O} \%$ \\
\hline Total & 107 & $25.8 \%$ \\
\hline
\end{tabular}

Fuente: Base de datos Proyecto: Caracterización psicosocial de las familias del Barrio 20 de JulioMunicipio de Urrao.

El 25.8\% de la población encuestada realiza en la actualidad algún nivel de formación, de la cual 15\% (63 personas) cursa estudios en básica primaria y el 10.8\% (44 personas) en secundaria. No se reportan personas realizando niveles técnicos, tecnológicos o universitarios (Tabla 3).

\subsubsection{Nivel Económico}

Gráfico 2: Distribución de personas que aportan económicamente para la manutención de la familia

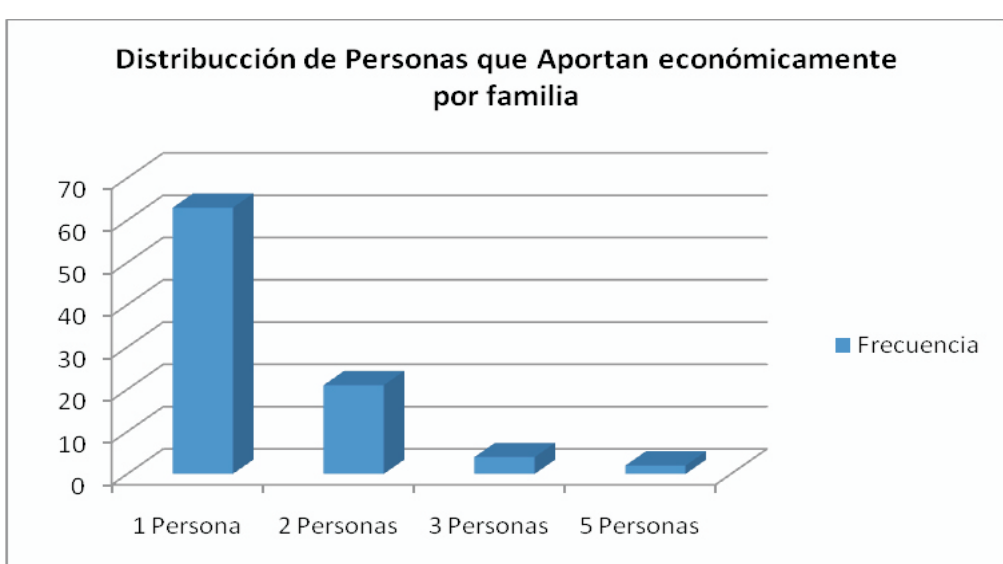

Fuente: Base de datos Proyecto: Caracterización psicosocial de las familias del Barrio 20 de Julio Municipio de Urrao. 
Sobre el número de personas que aportan económicamente para la manutención del hogar, se encuentra que en el 70\% (63 familias) de las familias una persona aporta económicamente, en el 23\% (21 familias) lo hacen dos miembros y en un $6 \%$ (6 familias) aportan más de 3 personas.

Las personas que son proveedoras económicamente en las familias se encuentra que: el $53 \%$ es el padre, el $41 \%$ las madres y el $22 \%$ los hijos sin que sean los responsables directos de la economía del hogar.

Gráfico 3. Distribución de ocupación laboral de los miembros de la familia.

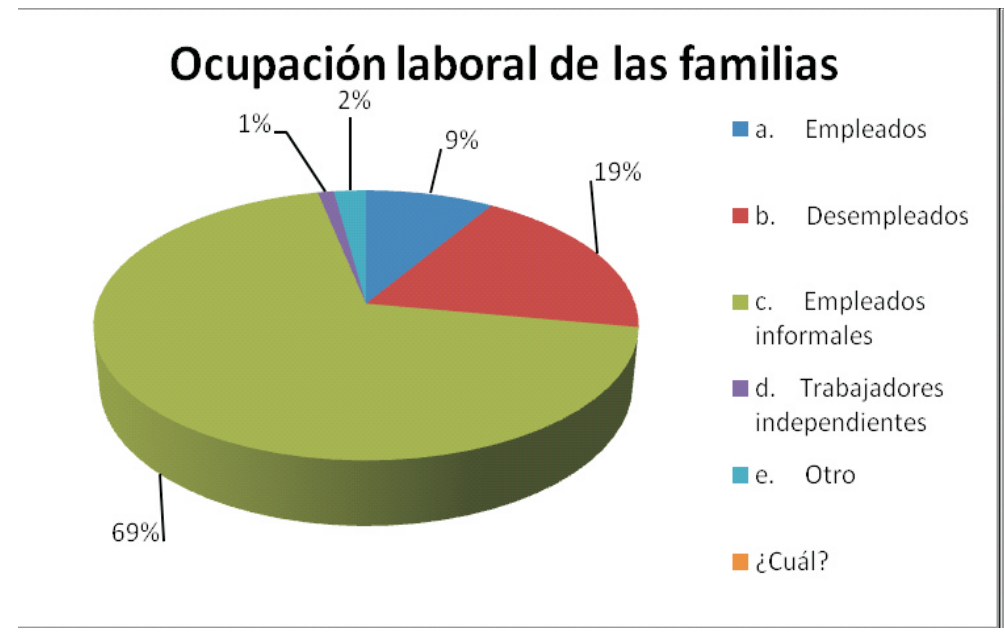

Con relación al tipo de vinculación laboral de las familias encuestadas, aproximadamente el $69 \%$ obtienen sus ingresos de oficios y ocupaciones informales relacionadas con la agricultura y labores afines como el jornaleo y en el caso de las mujeres labores domesticas en casas de familia. Existe una tasa de desempleo y desocupación del $19 \%$ y sólo el $9 \%$ tienen empleos formales con las garantías que esto implica, como estabilidad en los ingresos, servicios de salud, pensiones y riesgos entre otros. En el $2.2 \%$ de los casos los ingresos provienen de otras fuentes como pensiones y renta de intereses. 


\section{2 tiempo de residencia en el barrio, lugar de procedencia de la familia y razones que llevan a las familias a habitar el barrio}

En términos generales los habitantes del Barrio 20 de Julio son oriundos del Municipio de Urrao, sin embargo existen habitantes provenientes de Municipios cercanos de la subregión del suroeste de Antioquia, principalmente del municipio de Betulia.

\section{Tabla 4. Tiempo de residencia de las familias en el Barrio}

\begin{tabular}{|l|c|c|}
\hline \begin{tabular}{l|l|} 
Tiempo de residencia \\
en el barrio
\end{tabular} & Frecuencia & Porcentaje \\
\hline 5 años y menos & 17 & $18,89 \%$ \\
\hline de 6 a 10 años & 14 & $15,56 \%$ \\
\hline Entre 11 y 15 años & 10 & $11,11 \%$ \\
\hline Entre 16 y 20 años & 13 & $14,44 \%$ \\
\hline Mas de 20 años & 30 & $33,33 \%$ \\
\hline No responde & 6 & $6,67 \%$ \\
\hline Total & $\mathbf{9 0}$ & $\mathbf{1 0 0 , 0 0 \%}$ \\
\hline
\end{tabular}

Fuente: Base de datos Proyecto: Caracterización psicosocial de las familias del Barrio 20 de JulioMunicipio Urrao.

El tiempo de residencia en el barrio reportado por las familias, corresponde a un rango entre 1 y más de 20 años. Encontrándose que un 33\% de las familias llevan más de 20 años, seguido por quienes reportan una residencia entre cinco y menos años (19\%); y finalmente un $15 \%$ reportó vivir en él entre 6 y 10 años.

Las razones por las cuales las familias eligieron el Barrio 20 de Julio como lugar para vivir, están relacionadas con: La oportunidad de vivienda a bajos costos (tenencia de un terreno para construcción de vivienda), condiciones las laborales como oportunidad de ingresos económicos, y en algunos casos por desplazamiento forzado asociado a los fenómenos de violencia vividos en las diferentes regiones del país e incluso en las mismas veredas del Municipio de Urrao. Aunque se reportan 16 casos de familias en situación de desplazamiento, en la entrevista realizada a la rectora de la escuela del barrio, se evidencia que en el Centro Educativo Rural 20 de Julio, se encuentran 85 niños certificados con carta de población desplazada, con lo cual se infiere que en el barrio se asienta un porcentaje mayor de familias desplazadas. 


\subsection{Composición y dinámica familiar}

\subsubsection{Tipo de familia y dinámica familiar}

La tipología de las familias entrevistadas, o línea de parentesco que se establece entre los miembros que las componen, se caracterizan en un $51 \%$ como nuclear, el $26 \%$ de los hogares lo constituyen familias mono parentales constituidas principalmente por mujeres cabeza de familia. El 23\% restante corresponde a familias uniparentales (personas que viven solas), y a familias extensas.

\section{Gráfico 4. Percepción sobre las relaciones familiares}

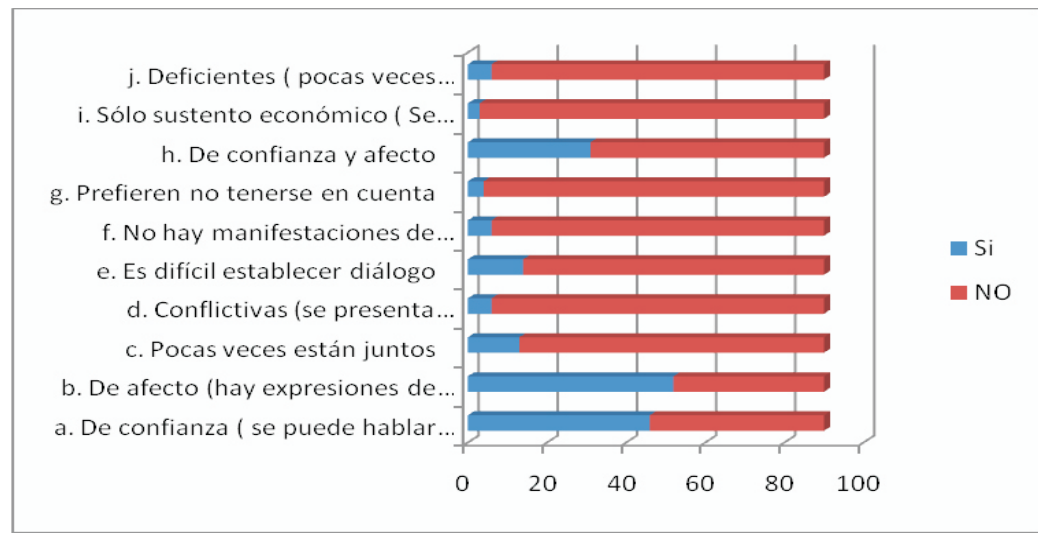

Fuente: Base de datos Proyecto: Caracterización psicosocial de las familias del Barrio 20 de Julio Municipio de Urrao.

En cuanto a la dinámica familiar, en general las relaciones entre sus miembros son valoradas como de confianza y afecto, no obstante, en la gráfica se estiman indicadores a través de los cuales pueden inferirse dificultades al interior de la familia, en cuanto a estrategias para la resolución de conflictos y optimización de la comunicación, así, el 7\% de los casos reportaron tener relaciones conflictivas (de maltrato); el 15.5\% manifestó dificultades en el establecimiento del diálogo; el $14 \%$ expresó el poco tiempo compartido con la familia, y el $4 \%$ prefieren no tenerla en cuenta. 
Tabla 5. Peleas verbales y físicas en la familia

\begin{tabular}{|l|c|c|c|c|}
\hline \multirow{2}{*}{ Opciones } & \multicolumn{2}{|c|}{ Peleas verbales } & \multicolumn{2}{c|}{ Peleas físicas } \\
\cline { 2 - 5 } & Frecuencia & Porcentaje & Frecuencia & Porcentaje \\
\hline $\mathrm{Si}$ & 31 & $34,4 \%$ & 10 & $11,1 \%$ \\
\hline No & 52 & $57,7 \%$ & 73 & $81,1 \%$ \\
\hline No aplica & 7 & $7,7 \%$ & 7 & $7,7 \%$ \\
\hline Total & $\mathbf{9 0}$ & $\mathbf{1 0 0 , 0 \%}$ & $\mathbf{9 0}$ & $\mathbf{1 0 0 , 0 \%}$ \\
\hline
\end{tabular}

Fuente: Base de datos Proyecto: Caracterización psicosocial de las familias del Barrio 20 de Julio -

La tabla 5 indica la presencia de maltrato intrafamiliar, expresado en sus formas verbales (34\%) y físicas (11\%).

\section{Tabla 6. Frecuencia mensual de peleas verbales y físicas en las familias}

\begin{tabular}{|l|c|c|c|c|}
\hline \multirow{2}{*}{\begin{tabular}{|} 
Tipo de pelea & \multicolumn{2}{|c|}{ Frecuencias al mes representadas en } \\
porcentajes
\end{tabular}} \\
\cline { 2 - 5 } & a. 1-3 & b. 4-6 & c. 7-9 & $\begin{array}{c}\text { d. más de } \\
\mathbf{1 0}\end{array}$ \\
\hline Verbales & $23.3 \%$ & $3.3 \%$ & $4.4 \%$ & $2.2 \%$ \\
\hline Físicas & $5.5 \%$ & $0 \%$ & $1.1 \%$ & $3.3 \%$ \\
\hline
\end{tabular}

Fuente: Base de datos Proyecto: Caracterización psicosocial de las familias del Barrio 20 de Julio Municipio de Urrao.

En relación con la frecuencia se encontró que en el $23 \%$ de las familias el maltrato verbal es utilizado de 1 a 3 veces al mes. En el caso del maltrato físico el $6 \%$ alcanzó una regularidad de 1 a 3 veces al mes, además el $3 \%$ reportaron intensidades que superan las 10 veces.

El maltrato verbal y físico, se presenta según la encuesta, con mayor frecuencia en la relación filial, seguido por la relación conyugal y con menor frecuencia en la relación paternal. 


\subsubsection{Manejo de normas y límites en el hogar}

\section{Tabla 7. Estilos parentales}

\begin{tabular}{|l|c|c|}
\hline \multicolumn{1}{|c|}{ Opciones de respuestas } & SI & NO \\
\cline { 2 - 3 } & Frec & Frec \\
\hline Sobreprotectores & 14 & 76 \\
\hline Autoritarios & 21 & 69 \\
\hline Permisivos & 7 & 83 \\
\hline Abandonantes & 0 & 90 \\
\hline Asertivos & 36 & 54 \\
\hline
\end{tabular}

Fuente: Base de datos Proyecto: Caracterización psicosocial de las familias del Barrio 20 de Julio - Municipio de Urrao.

Frente al manejo y establecimiento de normas y límites en el hogar, el $40 \%$ de las familias considera que tienen un manejo adecuado de la norma (asertivos), con sanciones alternas a la agresión física y/o psicológica. El 23\% asumen actitudes acordes a pautas autoritarias, el $16 \%$ de los hogares consideran ser sobreprotectores, y el $8 \%$ asumen actitudes permisivas, cifras que indican las dificultades que se presentan en la educación de los hijos.

Según la percepción de los adultos las mayores dificultades en la educación de los hijos, se presentan con los niños/as (39\%) y adolecentes (32\%), por falta de seguimiento a la norma y por problemas de adicción especialmente en los adolecentes.

\subsection{Condiciones de la vivienda en general}

\subsubsection{Tipo de vivienda y servicios públicos}

Tabla 8. Tipo de vivienda en la que habitan las familias del Barrio 20 de Julio

\begin{tabular}{|c|c|c|c|}
\hline Tipo de vivienda & Frecuencia & Porcentajes & Porcentajes acumulados \\
\hline Casa & 64 & $71,1 \%$ & $71,1 \%$ \\
\hline Apartamento & 1 & $1,1 \%$ & $72,2 \%$ \\
\hline Pieza & 7 & $7,8 \%$ & $80,0 \%$ \\
\hline Tugurio & 18 & $20,0 \%$ & $100,0 \%$ \\
\hline Total & 90 & $100,0 \%$ & $100,0 \%$ \\
\hline
\end{tabular}

Fuente: Base de datos Proyecto: Caracterización psicosocial de las familia Barrio 20 de Julio Municipio Urrao. 
La tabla 8 refleja los datos sobre el tipo de vivienda de las familias, así, el $71 \%$ reportó vivir en casa, el $20 \%$ tugurios construidos con tablas, plásticos y tejas de zinc; el $8 \%$ viven en piezas y un $1 \%$ en apartamento.

Sobre el tipo de tenencia de la vivienda, el $80 \%$ de las familias reportó que son propietarios lo cual lo sustentan con una compraventa, porque las propiedades no han sido legalizadas, y los terrenos se encuentran a nombre de la Junta de Acción Comunal. El 2\% reportan estar pagando la propiedad a la Junta de Acción Comunal, el 12\% manifiestan que la casa es prestada; y el 6\% paga canon de arrendamiento.

\section{Tabla 9. Número de dormitorios en las viviendas}

\begin{tabular}{|c|c|c|}
\hline Número de dormitorios & Frecuencias & Porcentajes \\
\hline 1 & 21 & $23,6 \%$ \\
\hline 2 & 42 & $47,2 \%$ \\
\hline 3 & 20 & $22,5 \%$ \\
\hline 4 & 7 & $6,7 \%$ \\
\hline Total & 90 & $100,0 \%$ \\
\hline
\end{tabular}

Fuente: Base de datos Proyecto: Caracterización psicosocial de las familias Barrio 20 de julio municipio Urrao.

En lo relacionado a la distribución de la vivienda la tabla 9 indica que el 47,2\% están constituidas por dos habitaciones, el $24 \%$ por una y el 23\% por tres; mientras que el $7 \%$ tienen 4 y mas habitaciones. Los datos anteriores reflejan las condicione de hacinamiento en el que viven las familias, si se tiene en cuenta que el número de habitantes por hogar es de 2 a 7 personas, es decir que el índice de ocupación por cada cama en un hogar es de 3.6.

\section{Gráfico 7. Servicios públicos y saneamiento básico de las viviendas}

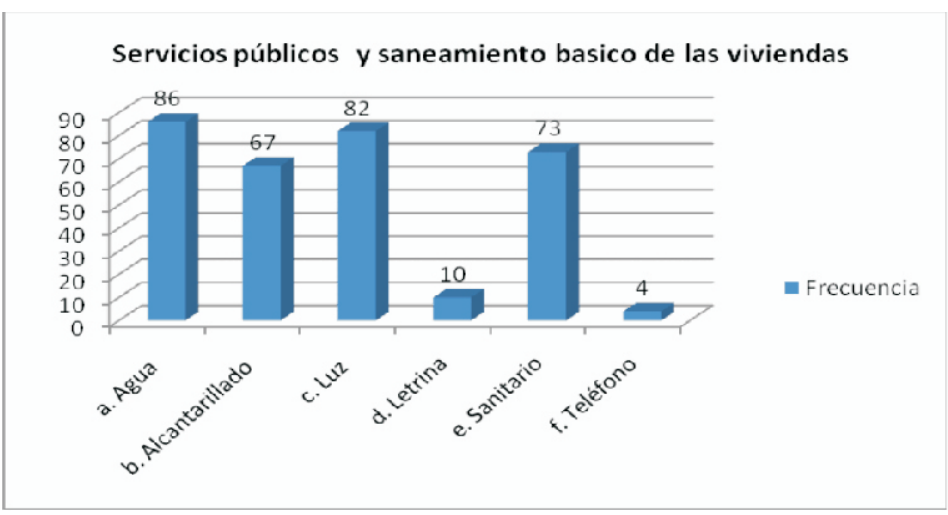

Fuente: Base de datos Proyecto: Caracterización psicosocial de las familias Barrio 20 de Julio Municipio Urrao. 
En servicios públicos, de acuerdo con los datos obtenidos en la encuesta, el $96 \%$ de las viviendas poseen acueducto, suministrado por la Junta de Acción Comunal y el Municipio. El $74 \%$ de las viviendas cuenta con servicio de alcantarillado, el $91 \%$ tiene energía eléctrica, el $81 \%$ posee inodoro, el $11 \%$ cuenta con letrinas y solo el $4 \%$ con servicio telefónico.

\subsection{Percepción de la familia sobre las problemáticas que afectan el barrio 20 de julio}

El presente apartado describe la percepción que tienen los habitantes con relación a las principales problemáticas que los afectan y los recursos que necesitarían para resolverlos.

\subsubsection{Principales problemáticas del Barrio 20 de Julio según sus habitantes:}

Las principales problemáticas expresadas por los habitantes del barrio se relacionan con:

3.5.1.1 Situaciones que afectan el equipamiento urbano, vivienda e infraestructura de la malla vial: Frente al equipamiento urbano se puede decir que en la actualidad el barrio no cuenta con parques y placas polideportivas, abiertas y de uso público, espacios necesarios para el esparcimiento, encuentro y recreación de la comunidad. Los jóvenes son quienes principalmente sienten esta necesidad, señalando que el deporte sería una manera de aprovechar el tiempo libre y prevenir el consumo de sustancias psicoactivas $y / o$ conductas delictivas.

Se observa además ausencia de andenes, pavimentación de calles, falta de iluminación pública, lo cual se convierte en un factor de inseguridad dentro del barrio. Otra necesidad es la red de lluvias, que afecta principalmente a la población en temporadas de invierno.

Las viviendas requieren de mejoramiento, pues aunque hay casas construidas en material y bareque, éstas se encuentran en mal estado, además, es característico encontrar tugurios construidos en tablas, plásticos y laminas de zinc. Algunas viviendas no cuentan con servicios públicos.

Para la comunidad es importante contar con la Caseta comunal puesto que en ella encuentran un espacio para la puesta en común de los asuntos de interés general barrial, el debate y las reuniones, aunque expresan que ésta requiere mejorar su estructura.

3.5. 1.2 Problemáticas relacionadas con el grupo familiar: La violencia intrafamiliar se convierte en una de las situaciones que tiene mayor impacto en las familias, la cual se expresa en sus 
formas físicas, verbal, psicológica y de abuso sexual. En esta situación se rescata el poco respeto hacia los niños/as, asociado a dificultades en la educación de los hijos por manejo inadecuado de normas y límites. Es de resaltar que la educación de los hijos es una inquietud decisiva en esta población, porque se emplean patrones de educación permisiva o autoritaria que tienen como consecuencia dificultades familiares y comunitarias. Además, la violencia conyugal se resalta como problemático.

Para esta población la falta de oportunidades para el empleo, y situaciones que para este contexto se relacionan con la pobreza como robos menores, la prostitución infantil en el barrio (auspiciada por los propios padres o hermanos) y fuera de él, el expendio y consumo de drogas no sólo por los habitantes del barrio, los bajos niveles nutricionales presentes en un importante porcentaje de personas en la comunidad y la mendicidad, también afectan la dinámica familiar y barrial.

3.5.1.3 Dificultades relacionadas con la convivencia entre vecinos: Uno de los aspectos señalados por la comunidad fue la desunión entre vecinos, falta de tolerancia, dificultades ocasionadas por la permisividad de algunos padres con sus hijos; las peleas por linderos de terrenos e irrespeto a la propiedad ajena, se convierten también en problemáticas. No son ajenos los altercados por distorsiones en la información o chismes, y por las estrategias inadecuadas para la resolución de conflictos.

3.5.1.4 Situaciones relacionadas con la educación y el empleo: En el tema de educación las personas perciben que hay pocas ofertas educativas para la validación de estudios de básica primaria, secundaria y media; además de ausencia de oportunidades de estudio para los jóvenes que terminan la secundaria. A nivel de oportunidades de empleo la población en general encuentran que es muy limitada.

3.5.1.5 Otros aspectos psicosociales que afectan la dinámica barrial: Pueden señalarse aspectos como: la falta de confianza en si mismos y "la pobreza de espíritu" como fue señalado por la comunidad en alguna de las ocasiones; los hurtos por ladrones del barrio o de otros barrios; la drogadicción se convierte en un problema que afecta a toda la comunidad no solo por los efectos que ocasiona a nivel personal sino que con ello se asocia el robo y algunas amenazas a personas del barrio; los expendios de drogas ilegales; el alcoholismo en los menores de edad, el mal manejo de la sexualidad asociado a la falta de educación y orientación; la prostitución infantil aceptada por los padres (asociada a la subsistencia de grupo familiar) según entrevistas con la comunidad; la ausencia de transporte público desde y hacia el barrio; la mendicidad en niños y adultos; la movilidad de las personas del barrio a causa del desplazamiento forzado y las incursiones de los grupos armados.

AGO.USB Medellin-Colombia V. $9 \quad N^{\circ} 1$ PP. 1-294 Enero-Junio 2009 ISSN: 1657-8031 


\subsection{Recursos que según la comunidad del barrio 20 de julio se requieren para resolver sus problemáticas}

Las familias consideran que la manera de resolver las dificultades en su comunidad guarda relación con la unión y el trabajo en equipo proveniente de ellos, no obstante, consideran que sus condiciones pueden ser transformadas si existe mayor presencia de la institucionalidad y se mejorara el acceso a los mecanismos de justicia y beneficios ofrecidos por ésta. Como acciones específicas que se esperarían de la institucionalidad se rescatan las siguientes:

Mayor presencia de las autoridades competentes para la regulación y el manejo de expendios de drogas y otras situaciones que requieren de este tipo de control como los robos y la prostitución infantil

Creación de iniciativas de gestión y autogestión de ingresos, de ofertas de empleo dirigidas a la población barrial

Mejoramiento de vivienda, pavimentación calles, creación de la red de lluvias y zonas para la recreación y el deporte

Mejoramiento en ofertas educativas y de capacitación para la población

Intervención y capacitación a la comunidad en aspectos que contribuyan a la disminución y prevención de problemáticas como la violencia intrafamiliar, el consumo de sustancias psicoactivas y la prostitución infantil.

\section{Discusión y conclusiones}

Teniendo en cuenta que la familia es un espacio de socialización primaria donde la personas deben recibir protección y los primeros cimientos para relacionarse con su cultura, entorno y sociedad, resulta oportuno concluir que existen múltiples factores de riesgo internos y externos a las familias del Barrio 20 de Julio, los cuales pueden afectar dicha tarea y con ello el desarrollo de habilidades personales para la convivencia. De esta manera, se hace oportuno plantear una estrategia de intervención para fortalecer los factores protectores y disminuir los factores de riesgo identificados en la investigación, apoyando la resignificación de los estilos relacionales de ésta población. A continuación se discute la incidencia de algunos de los factores que pueden afectar la convivencia. 


\subsection{Situación educativa en la población}

La educación se convierte en uno de los atributos principales a través del cual se espera una contribución al pleno desarrollo de la personalidad y la calidad de vida de las personas, puesto que, está relacionada con una formación oportuna para el desarrollo de competencias laborales necesarias para la inclusión en el mundo laboral y la posibilidad de mejorar ingresos económicos que contribuyan a la satisfacción de necesidades básicas individuales y del grupo familiar. Por esta razón para el Estado Colombiano en las últimas décadas una de sus prioridades ha sido garantizar la gratuidad de la educación en básica primaria, secundaria y media vocacional, no obstante, el problema de cobertura educativa persiste cuando no existen otras condiciones conexas, como las económicas (por ejemplo para el desplazamiento de estudiantes desde sus hogares al sitio de estudio), motivacionales en los niños, adolescentes y adultos para vincularse a procesos educativos. De la mano con lo anterior, se encuentra escasa motivación en los docentes, temores por su seguridad y escasa preparación o actualización frente a nuevos retos pedagógicos; se encuentra también precariedad en recursos didácticos e infraestructurales para enfrentar la realidad local, regional y global, y para que sea más universal el acceso de la población a tal servicio.

De acuerdo con lo anterior, el Barrio 20 de Julio no está exento de estas condiciones, así, aún con los avances de cobertura educativa en el Municipio, persiste en el barrio como problemática la alta deserción escolar en el nivel de básica primaria y secundaria, en este sentido, la educación deja de ser un factor protector para los niños/as y jóvenes del barrio que desertan por diferentes situaciones, entre ellas, por apoyar la manutención del hogar, responder a las necesidades del padre/madre que se desplaza entre municipios y veredas como jornalero en las diferentes temporadas de cosecha, la falta de coherencia entre las propuestas educativas y las posibilidades de desempeño de los niños/as y jóvenes, así como la poca preparación de docentes y apoyo a los mismos para enfrentar diferentes problemáticas en el aprendizaje de los estudiantes.

Debe tenerse en cuenta, con relación a la educación, que así mismo, una vez finalizados los estudios de secundaria, las pocas posibilidades y ofertas para la incursión en estudios técnicos, tecnológicos y profesionales se convierten en factores que limitan el desarrollo integral de los jóvenes, quienes al no contar con las oportunidades necesarias para dar continuidad a sus estudios, se quedan en su barrio exponiéndose a situaciones de riesgo frente a problemáticas como el desempleo, el consumo de sustancias psicoactivas, o la inserción a grupos armados y/o delincuenciales, quienes garantizan ingresos económicos para beneficio individual y/o apoyar a sus familias. Todas estas situaciones, disminuyen las posibilidades de preparación de ésta población para el mundo laboral y con ello se perpetúa la pobreza, las bajas condiciones 
en la calidad de vida, y se convierten en contribuyentes para el deterioro en la convivencia familiar y social.

Otro elemento a considerar frente a lo educativo, son los bajos niveles en la escolaridad de los padres/madres o tutores del hogar, los cuales inciden en los recursos con los que puedan contar para orientar la formación de niños/as y jóvenes; tales bajos niveles educativos terminan siendo un predictor del uso de la agresión como una manera de educar, aunque es de clarificar, que no todo padre con bajos niveles de escolaridad recurre a la agresión como pauta de educación.

Es de rescatar además que dentro del porcentaje de la población en edad no escolar (16.3\%), se encontraron casos en los cuales, los niños podrían estar en el grado preescolar, no obstante, no se encuentran incluidos porque según la percepción de los padres o tutores, el niño/a no contaban con la edad suficiente para estar incluido en el sistema educativo.

Al respecto, el Municipio está llamado a posibilitar el acceso efectivo a una enseñanza de calidad para niños, jóvenes (concentrando esfuerzos en la vinculación de aquellos que están en extraedad escolar) y los adultos, brindando una enseñanza básica, media y superior, que posibilite no sólo una adecuada calificación de recursos humanos para incorporarse al proceso productivo, sino también para la formación de ciudadanos activos y responsables en el ejercicio de sus derechos y deberes.

\subsection{Condiciones económicas de la población}

Respecto a las precarias condiciones económicas y laborales de la población, se puede señalar que es un factor que vulnera diferentes derechos de la población, y, con ello se pueden detonar situaciones que afectan la convivencia pacifica. Así, la inestabilidad a nivel laboral y los bajos ingresos, se convierten en situaciones que contribuyen a la insatisfacción de necesidades básicas como la alimentación, la vivienda digna, la salud, la recreación y la educación. Tal precariedad económica, además pone en riesgo la convivencia familiar y comunitaria por las condiciones de hacinamiento, mendicidad, robo, venta de drogas y prostitución infantil, con las que puede ser asociada. Por otra parte, facilita que la población considere la vinculación a grupos armados, como una posibilidad de subsanar su situación económica; además de condiciones como el estrés que se convierte en problemático cuando las circunstancias desbordan la capacidad de afrontamiento de la persona, detonando la aparición de comportamientos agresivos para resolver los problemas de educación con los hijos, las dificultades de pareja y entre vecinos.

En el caso de las mujeres cabeza de familia, las condiciones laborales y económicas se convierten en una situación problemática; mientras su interés principal se centra en la 
satisfacción de las necesidades básicas de su familia, aspectos relacionados con la educación de los hijos, quedan desprotegidos por la falta de tiempo para compartir con éstos, o para establecer un control desde las normas y limites en el hogar. No es raro escuchar entonces en la voz de las persona de la comunidad, sobre los niños que no permanecen bajo la tutela de padres o adultos responsables, y quedan por fuera del hogar (" ...que esos niños... permanecen haciendo y deshaciendo ${ }^{7} . . . "$ o de "... son niños que no tienen ley...").

Es importante tener en cuenta que dadas las condiciones laborales de inestabilidad, irregularidad y de desempleo, y las características de la población (campesinos) es importante pensar en iniciativas como: proyectos productivos auto-sostenibles que contribuyan, por una lado, al fortalecimiento de lazos solidarios por parte de la comunidad, y, por otro, a la generación de ingresos a la economía familiar, mejorando las condiciones de vida de sus habitantes, al generarse nuevos ingresos y en donde podría participar toda la familia, colocándolos en situaciones más equitativas y dignas como seres humanos.

\subsection{Problemáticas que afectan las relaciones familiares}

Frente a las problemáticas que afectan la dinámica familiar, es de considerar en primer lugar que dentro de la vivencia de su historia, las familias han atravesado por situaciones de guerra que les ha obligado a desplazarse hasta el barrio, o que, de igual manera han padecido en su sector la presencia de grupos armas como el frente 34 de las FARC y las AUC. Estas situaciones se han convertido en factores que han intervenido en la intimidad familiar ya sea por la pertenencia de algunos miembros de las familias a estos grupos y por los duelos sin elaborar de las familias que perdieron seres queridos a causa de la "limpieza social", convirtiendo el barrio en un sector de mayor vulnerabilidad por las diferentes problemáticas con las que llegan las familias, no solo a nivel relacional, sino a nivel económico. Además, estas condiciones aunque reales, generan también una estigmatización ante el resto de habitantes del municipio, operando en dos sentidos, por un lado, cierra posibilidades a las familias del barrio, y, por otro, los mismos habitantes del sector interiorizan dicha estigmatización asumiendo actitudes que nos les permite gestionar las propias posibilidades y condiciones de inclusión.

Es de considerar, además, que la guerra vivenciada por las familias, se convierte en un factor predisponente para la violencia intrafamiliar, así aunque un porcentaje significativo de la población reportó tener unas adecuadas relaciones familiares y manejo de la norma y la autoridad, un porcentaje igualmente significativo reporta que en su familia existen indicadores de violencia intrafamiliar presentada en todas sus formas (física, psicología y sexual) y en todos los lazos afectivos (conyugal, filial y paterno filial). 
Se reportaron además dificultades en la educación de los hijos asociadas a pautas parentales permisivas y autoritarias, la primera, porque no contribuye a que los niños/ñas y jóvenes construyan las normas y limites necesarios para la vivencia en comunidad, y la segunda, porque las representaciones sociales y creencias de aceptación del uso de comportamientos agresivos para normatizar y corregir a los hijos, autoriza que los padres/madres los maltraten con el propósito de educarlos, y con ello, se perpetua la violencia como una estrategia para resolver las crisis que ocurren al interior de la familia y se da un aprendizaje que puede ser extrapolado al mundo social.

\subsection{Calidad de la vivienda}

Dentro de las condiciones que afectan a las familias y sus dinámicas, la calidad en la vivienda es un factor que de acuerdo con pacto Internacional sobre Derechos Económicos, Sociales y Culturales, es uno de lo más violados, pues un porcentaje significativo de la población se encuentra en condiciones de vulnerabilidad, ante la falta de una vivienda digna que ofrezca las condiciones físicas, de espacio, servicios públicos adecuados y legalidad. Por lo anterior, es necesario para esta población crear espacios y proyectos de gestión direccionados con el fin de obtener los recursos necesarios para el mejoramiento de vivienda y legalización de predios, expansión y crecimiento urbanístico, aprovechamiento adecuado de los recursos hídricos y del medio ambiente.

\subsection{Otras problemáticas que afectan la salud mental y la convivencia}

En cuanto a los asuntos que afectan la salud mental y física, problemáticas como el hurto, expendio y consumo de sustancias psicoactivas, la iniciación temprana en la sexualidad con el creciente riesgo de contagio de enfermedades de transmisión sexual, el maltrato físico y psicológico, son problemáticas que requieren de intervención multiestamental, puesto que repercuten directamente en la vida social del barrio.

Para la atención a esta población, es importante advertir que si bien en el plan de Desarrollo Municipal están contemplado unos lineamientos claros de intervención (desarrollo integral), estos, aún no se ven reflejados en el barrio, lo cual puede explicarse por factores como, procesos propios de ajuste y empalme gubernamental, que se espera sean subsanados pronto en la gestión de la Administración actual, o, aún existiendo voluntad política, se carece de los medios e instrumentos necesarios para la ejecución de proyectos y programas. Frente a fenómenos sociales como los que se presentan en el Barrio 20 de Julio, es necesario trabajar desde el concepto de red, en este caso redes interinstitucionales y sociales que trabajen de manera coordinada, posibilitando acciones menos aisladas y de mayor impacto para la población. 
De esta manera, para lograr verdaderos cambios es necesario que haya una continuidad en el tiempo con programas definidos desde el diálogo Institución-Comunidad, ComunidadInstitución y que apunten a la cobertura de las necesidades básicas insatisfechas. En igual medida es tarea de las instituciones dar a conocer a las comunidades sus diferentes programas de tal manera que estas dejen de ser actores pasivos y se conviertan en actores participativos de los diferentes procesos y decisiones barriales o comunitarias.

Sobre los factores que pueden favorecer la intervención, se rescata el potencial dentro de esta población que pasa por adolescentes, jóvenes, mujeres cabeza de familia, hombres agricultores y adultos mayores, potencial que hay que hacer visible para sí mismos (que la gente crea que si es posible realizar cambios) y para el resto de la población. Se observa, además el reconocimiento que tiene la Secretaría de Bienestar social en la comunidad, el cual se percibe como credibilidad instifucional $y$, desde tal dependencia se puedan plantear acciones que permitan el trabajo en red. 


\section{Referencias Bibliográficas}

Amar Amar, J. J. (2000). Niños invulnerables: factores cotidianos de protección que favorecen el desarrollo de los niños que viven en contextos de pobreza. EN: Psicología desde el Caribe/No.5 (p.96-126)

Baumrind, D. (1989). Rearing competent children. En W. Damon (Ed.), Child development today and tomorrow (pp.349 - 378). San Francisco: Jossey-Basss Baumrind, D. (1991). The influence of parenting style on adolescent competence and substance use. Journal of Early Adolescence, 1 1, 56-95.

Bowlby, J. (1993). El Vínculo Afectivo. España: Paidos.

Buendía, J. (1999). Familia y psicología de la salud España: Pirámide.

Coloma, J. (1993). La familia como ámbito de socialización de los hijos. EN: Pedagogía familiar. Madrid: J.M. Quintana (Ed.)

Concejo de Medellín (1994). Foro Nacional de la Familia - 1993. Colombia

Esteve Rodrigo J.V. (2004). Estilos parentales, clima familiar, y autoestima física en adolescentes. Tesis Doctoral. Universitat de València. Facultad de Psicología. Proyecto de investigación SEJ2004-01742 financiado por el Ministerio de Educación y Ciencia. España.

Estrada, A.M., Ibarra, C. \& Sarmiento, E. (2003). Regulación y control de la subjetividad y la vida privada en el contexto conflicto armado colombiano. EN: Revista de Estudios Sociales. Bogotá, Universidad de Los Andes, No. 15, 133- 149.

Hernández, Á. (1997). Familia, ciclo vital y psicoterapia. Sistémica breve

Kotliarenco, M. A. (2000). La caracterización de la familia en condiciones de pobreza: su aporte a la educación inicial. EN: Psicología desde el Caribe No.5 (p.373-394).

Musitu, G. \& Molpeceres, M.A. (1992). Estilos de socialización, familismo y valores. Infancia y Sociedad, 16, 67-101. 
Musitu, G., Moliner, M. García, F., Molpeceres, M.A., Lila, M.S. \& Benedito, M.A. (1994). Estilos de socialización parental y percepción del adolescente: Exploración de sus relaciones en una muestra polaca. En G. MUSITU (Dir.): Intervención comunitaria. (pp. 143-156). Valencia: Cristóbal Serrano Villalba.

Quintero Velásquez, A.M. (1997). Trabajo Social y proceso familiares. Buenos Aires: Lumen Humanitas. Pág. 187

Reimers, F. (1999). Oportunidades educativas para las familias de escasos recursos en America Latina. EN: Perspectivas (Unesco) V29/No.4 (p.595-611).

Riso, W. (2003). Familia y convivencia. Colombia: Periódicos Asociados

Rubio, M. (1997). Los costos de la violencia en Colombia. Bogotá, Documento CEDE, No. 10, diciembre, Uniandes.

Simón, M. I.; Triana Pérez, B.; Camacho, J. (2001). La construcción del concepto de familia: de las concepciones implícitas a las explicitas. EN: Infancia y aprendizaje. (P.425-439)

Terre Des Hemmes Suisse y Universidad de San Buenaventura Medellín (2008). Investigación factores causales de explotación sexual comercial en niños, niñas y adolescentes en la Costa Atlántica, Municipios de Barranquilla y Sincelejo, con especial énfasis en la población desplazada por la violencia. Medellín

UNICEF (2001). Ciudades para la niñez. Madrid: Oficina Regional para América Latina y el Caribe de UNICEF (TACRO).

Urrutia, N. (2004). El gasto en defensa y seguridad: caracterización del caso colombiano en el contexto internacional. Bogotá, Archivos de Economía, Documento 249, Departamento Nacional de Planeación.

${ }^{6}$ Por factores protectores se comprende aquellas características que permiten a los seres humanos o comunidades resistir traumatismos violentos y transformarlos; por factores de riesgo se entienden aquellas características que les hacen vulnerables para el estrés disminuyendo las posibilidades de afrontamiento a dichos traumatismos.

${ }^{7}$ Fuente: Informes cualitativos entrevistas y conversatorios realizados con la comunidad del Barrio 20 de Julio de Urrao-Antioquia, 2008. 